\title{
Improvement of adynamic bone disease after renal transplantation
}

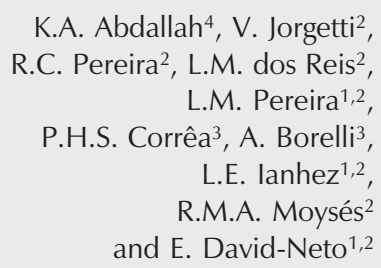

\author{
Unidade de Transplante Renal, 'Divisão de Urologia, \\ ${ }^{2}$ Nefrologia, ${ }^{3}$ Endocrinologia, ${ }^{4}$ Alergia e Imunologia Clínica, \\ Hospital das Clínicas, Faculdade de Medicina, Universidade de São Paulo, \\ São Paulo, SP, Brasil
}

\begin{abstract}
Correspondence
E. David-Neto

Unidade de Transplante Renal

Hospital das Clínicas, FM, USP

Rua Ferdinando Laboriau, 263

01250-040 São Paulo, SP

Brasil

E-mail: elias.david.neto@attglobal.net

Research supported by FAPESP

(No. 95/02431-9). R.M.A. Moysés

was also supported by FAPESP

(No. 98/06352-4).

$\ldots \ldots \ldots \ldots \ldots \ldots \ldots \ldots$
\end{abstract}

Received December 1, 2004

Accepted August 19, 2005

\begin{abstract}
Low bone remodeling and relatively low serum parathyroid hormone (PTH) levels characterize adynamic bone disease (ABD). The impact of renal transplantation (RT) on the course of $\mathrm{ABD}$ is unknown. We studied prospectively 13 patients with biopsy-proven ABD after RT. Bone histomorphometry and bone mineral density (BMD) measurements were performed in the 1st and 12th months after RT. Serum PTH, 25-hydroxyvitamin D, 1,25-dihydroxyvitamin D, and osteocalcin were measured regularly throughout the study. Serum PTH levels were slightly elevated at transplantation, normalized at the end of the third month and remained stable thereafter. Bone biopsies performed in the first month after RT revealed low bone turnover in all patients, with positive bone aluminum staining in 5. In the 12th month, second biopsies were performed on 12 patients. Bone histomorphometric dynamic parameters improved in 9 and were completely normalized in 6, whereas no bone mineralization was detected in 3 of these 12 patients. At 12 months post-RT, no bone aluminum was detected in any patient. We also found a decrease in lumbar BMD and an increase in femoral BMD. Patients suffering from ABD, even those with a reduction in PTH levels, may present partial or complete recovery of bone turnover after successful renal transplantation. However, it is not possible to positively identify the mechanisms responsible for the improvement. Identifying these mechanisms should lead to a better understanding of the physiopathology of $\mathrm{ABD}$ and to the development of more effective treatments.
\end{abstract}

\section{Introduction}

Renal transplantation (RT) typically corrects most mineral metabolism abnormalities that can lead to renal osteodystrophy. However, pre-existing bone diseases may not be completely resolved by RT, especially if the recovery of renal function is not
Key words

- Adynamic bone disease

- Aluminum bone disease

- Bone biopsy

- Parathormone

- Renal osteodystrophy

- Renal transplantation 
ies (1). In some cases, bone loss has been as high as $10 \%$. However, these studies were not able to determine the various patterns of renal osteodystrophy (high or low bone turnover) because they were based on BMD findings. Currently, renal osteodystrophy is differentiated solely by means of a bone biopsy and histomorphometric analysis (2) and few prospective studies have evaluated this parameter (3-6). For example, mild to moderate hyperparathyroidism is spontaneously reversed during the first 1 or 2 years after a successful RT (3). We have previously demonstrated that successful kidney transplantation clears the aluminum on the mineralizing surface and restores normal bone remodeling in patients with aluminumrelated osteomalacia (4). However, there are no data regarding the outcome of adynamic bone disease $(\mathrm{ABD})$ following kidney transplantation.

In the present study, we have examined the natural course of ABD after kidney transplantation and the effect of previous low bone remodeling activity on bone mass.

\section{Material and Methods}

\section{Patients and study design}

In order to identify patients with $\mathrm{ABD}$, all patients who lacked clear signs of moderate or severe hyperparathyroidism (parathyroid hormone $(\mathrm{PTH})>800 \mathrm{pg} / \mathrm{mL}$ or radiologic features of hyperparathyroidism) at admission for RT were considered. The study was approved by the Hospital Ethics Committee and only those who agreed to participate in the study were enrolled. After giving informed written consent, the patients were submitted to a bone biopsy during the first month after transplantation. The 13 patients in whom $\mathrm{ABD}$ was confirmed remained in the study.

The BMD of the lumbar spine (L1-L4) and of the total femoral neck was also measured. After following the patients for 1 year,
BMD and bone biopsies were repeated. Hormonal and biochemical markers related to bone metabolism were routinely measured. Signs and symptoms related to bone disease were investigated.

\section{Laboratory measurements}

The first blood sample obtained from patients undergoing cadaver kidney transplantation was collected immediately prior to surgery. All other blood samples were collected in the morning after an overnight fast. Serum calcium (normal range $=9.0$ $11.0 \mathrm{mg} / \mathrm{dL}$ ), phosphorus (normal range $=$ 2.3-4.6 mg/dL), alkaline phosphatase (normal range $=60-170 \mathrm{U} / \mathrm{L}$ ), and creatinine (normal range $<1.4 \mathrm{mg} / \mathrm{dL}$ ) were measured with a Cobas-Integra autoanalyzer (Roche Diagnostics Corporation, Indianapolis, IN, USA). Osteocalcin (normal range $=6-37 \mathrm{pg} /$ $\mathrm{mL}$ ) and intact PTH (normal range $=8-76$ $\mathrm{pg} / \mathrm{mL}$ ) were measured by radioimmunoassay using Cis-Bio assays (CIS-BIO International, Gif-sur-Yvette, France). Levels of 1,25-dihydroxyvitamin $\mathrm{D}\left(1,25-(\mathrm{OH})_{2} \mathrm{D}_{3}\right.$, normal range $=16-55 \mathrm{pg} / \mathrm{mL}$ ) were determined by radioimmunoassay (Nichols Institute, San Juan Capistrano, CA, USA). Radioimmunometric assay (INCSTAR, Rohm and Haas Company, Dietzenbach, Germany) was used to measure 25-hydroxyvitamin D $\left(25-(\mathrm{OH}) \mathrm{D}_{3}\right.$, normal range $\left.=16-74 \mathrm{ng} / \mathrm{mL}\right)$. Blood samples were collected and kept at $20^{\circ} \mathrm{C}$ until analysis. Analysis was carried out in duplicate.

\section{Bone mineral densitometry}

Vertebral and femoral bone density was measured by dual-energy X-ray absorptiometry (Hologic QDR-4500, Waltham, MA, USA). The results are reported as BMD (g/ $\mathrm{cm}^{2}$ ) and as percentage of the value for an age- and sex-matched population. For serial measurements, the position and the region of interest were carefully determined. The 
coefficient of variation for a phantom spine evaluation was $0.5 \%$.

\section{Bone histomorphometry}

Biopsy specimens were obtained from the anterior superior iliac crest after double labeling with tetracycline, with a 10-day interval between doses. The bone specimens were fixed in $70 \%$ ethanol and processed for histomorphometry as previously described (7). For aluminum quantification, the material was stained with aurin tricarboxylic acid. Histomorphometric analysis of trabecular bone was performed using a single-blind manual system. Histomorphometric parameters in trabecular bone were measured according to standards established by Parfitt et al. (8). Patients with ABD were defined as those who exhibited a bone formation rate (BFR) lower than $0.031 \mu \mathrm{m}^{3}\left(\mu \mathrm{m}^{2}\right)^{-1}$ day $^{-1}$ (which is the lowest reported value in normal individuals) (9) along with an osteoid volume/bone volume ratio lower than $15 \%$, in the presence or absence of positive aluminum staining. Normal static histomorphometric parameters were established by postmortem examination of 94 disease-free individuals from the greater metropolitan area of the city of São Paulo, SP, Brazil. The BFR was calculated as the sum of tetracycline double-labeled surfaces plus half of the single-labeled surfaces times the mineral apposition rate. Dynamic histomorphometric parameter values were considered to be normal when within the range reported by Melsen and Mosekilde (10).

\section{Statistical analysis}

Changes with time were evaluated by repeated one-way ANOVA. Tukey's test was used to separate the group or groups of variables that differed from the others during ANOVA. Multiple logistic regression analysis was performed to identify factors that could predict a future event (such as improvement or no improvement in BFR). Fisher's exact test was used to compare event proportions. Values of $\mathrm{P}<0.05$ were considered to be statistically significant. Data are reported as means $\pm \mathrm{SD}$.

\section{Results}

\section{Patients}

The group studied consisted of 4 men and 9 women with a mean age of $42 \pm 8$ years (range 27-57). There were 9 Caucasians, 2 Afro-Brazilians, and 2 Orientals. Causes of end-stage renal disease were: chronic glomerulonephritis in 9 patients, chronic interstitial nephritis in 1, adult dominant polycystic kidney disease in 1 , and unknown in 2 . Twelve patients had been submitted to dialysis for an average of $31 \pm 24$ months prior to RT; 1 was under continuous ambulatory peritoneal dialysis and 11 were being treated with intermittent hemodialysis.

Before RT, 10 patients had received oral calcitriol for a mean of $19 \pm 24$ months, 4 had used oral aluminum hydroxide for a mean of $0.8 \pm 1.8$ months and 11 were treated with oral calcium carbonate for a mean of $20 \pm 20$ months.

Six patients received live donor kidneys and 7 received cadaveric kidneys. They were all treated with the classic triple therapy of prednisone, azathioprine and cyclosporin A. The initial oral dose of prednisone was $1 \mathrm{mg}$ $\mathrm{kg}^{-1}$ day $^{-1}$ and was progressively reduced to $0.2 \mathrm{mg} \mathrm{kg}^{-1}$ day $^{-1}$ in the third month and to $0.15 \mathrm{mg} \mathrm{kg}^{-1}$ day $^{-1}$ by the 12th month after RT. The azathioprine dose was $2 \mathrm{mg} \mathrm{kg}^{-1}$ day $^{-1}$ and was adjusted according to leukocyte count and liver enzyme activity. Cyclosporin A was started at 7-8 $\mathrm{mg} \mathrm{kg}^{-1} \mathrm{day}^{-1}$ and adjusted depending on trough levels.

The patients experienced a mean of $1.3 \pm$ 0.8 acute rejection episodes during the 1year follow-up period. Rejection episodes were treated with intravenous methylprednisolone, 8 or $16 \mathrm{mg} \mathrm{kg}^{-1} \mathrm{day}^{-1}$, for 3 con- 
secutive days as part of another ongoing study at our center. During this period, the mean cumulative prednisone dose was $9.8 \pm$ $2.5 \mathrm{~g}$. None of the patients studied presented steroid-resistant rejection episodes.

Renal function remained stable throughout the first year. Serum creatinine at the end of months $1,3,6$, and 12 was $1.9 \pm 0.9,1.5$ $\pm 0.4,1.5 \pm 0.3$, and $1.6 \pm 0.5 \mathrm{mg} / \mathrm{dL}$, respectively $(\mathrm{P}=\mathrm{NS})$. None of the patients rejected their grafts during the first year. Two patients presented progressive loss of renal function, but ended the first year with creatinine clearance above $30 \mathrm{~mL} \mathrm{~min}^{-1} 1.73\left(\mathrm{~m}^{2}\right)^{-1}$.

No oral calcium or phosphorus supplementation, diuretic therapy or anticonvulsants were administered after RT. At the end of the first year, mean body weight increased from $61.3 \pm 10.3$ to $68.0 \pm 15.2 \mathrm{~kg}(\mathrm{P}<0.05)$ and mean serum albumin levels increased from $3.7 \pm 0.5$ to $4.2 \pm 0.6 \mathrm{~g} / \mathrm{dL}(\mathrm{P}<0.05)$.

\section{Hormonal and biochemical parameters}

Table 1 shows the mean values of total serum calcium, phosphorous, alkaline phosphatase, and serum creatinine before and after RT. There were no changes in mean total serum calcium levels during the study period ( $\mathrm{P}>0.05)$. No patient developed hyper- or hypocalcemia. Mean phosphorous levels were higher before transplantation than at any point after transplantation. However, when mean phosphorous levels measured at the end of the first month were analyzed and compared with subsequent measurements,

Table 1. Serum total calcium, phosphorus, total alkaline phosphatase, and creatinine values at renal transplant (0) and 12 months later (12).

\begin{tabular}{|c|c|c|c|c|c|c|c|c|}
\hline & \multicolumn{2}{|c|}{$\mathrm{Ca}$} & \multicolumn{2}{|c|}{$P$} & \multicolumn{2}{|c|}{ TAP } & \multicolumn{2}{|c|}{$\mathrm{Cr}$} \\
\hline & 0 & 12 & 0 & 12 & 0 & 12 & 0 & 12 \\
\hline All $(N=13)^{a}$ & $8.8 \pm 0.9$ & $9.5 \pm 0.5$ & $6.3 \pm 1.6$ & $3.8 \pm 0.7^{*}$ & $183 \pm 150$ & $134 \pm 70^{*}$ & $10.0 \pm 3.6$ & $1.5 \pm 0.5^{\star}$ \\
\hline Same $(N=3)$ & $10.0 \pm 0.2$ & $9.6 \pm 0.6$ & $5.7 \pm 1.3$ & $4.1 \pm 0.5$ & $131 \pm 30$ & $115 \pm 16$ & $6.6 \pm 2.7$ & $1.4 \pm 0.2$ \\
\hline Better $(\mathrm{N}=3)$ & $9.0 \pm 0.5$ & $9.5 \pm 0.5$ & $7.2 \pm 2.4$ & $3.7 \pm 1.0$ & $247 \pm 216$ & $92 \pm 32$ & $11.5 \pm 4$ & $1.6 \pm 0.2$ \\
\hline Recovered $(\mathrm{N}=6)$ & $8.1 \pm 0.5$ & $9.4 \pm 0.5$ & $6.3 \pm 1.5$ & $3.9 \pm 0.9$ & $117 \pm 44$ & $128 \pm 53$ & $10.4 \pm 4$ & $1.7 \pm 0.8$ \\
\hline
\end{tabular}

The patients were divided into three groups: those who presented no improvement in bone formation rate (same), those who presented partial reversal of adynamic bone disease ( $A B D$; better), and those in whom $A B D$ was cured (recovered). Data are reported as means $\pm S D$. Ca $=$ total serum calcium (normal range $=9-11 \mathrm{mg} / \mathrm{dL}$ ); $\mathrm{P}=$ serum phosphorus (normal range $=2.3-4.6 \mathrm{mg} / \mathrm{dL}$ ); TAP $=$ total alkaline phosphatase $(\mathrm{normal}$ range $=60-170 \mathrm{U} / \mathrm{L}) ; \mathrm{Cr}=$ serum creatinine (normal range $<1.4 \mathrm{mg} / \mathrm{dL}$ ).

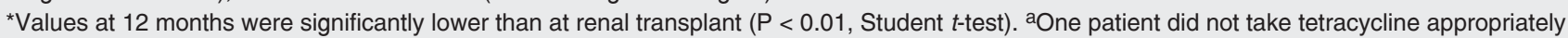
prior to the second biopsy; thus, analysis of improvement at 12 months was done for only 12 patients.

Table 2. Serum intact PTH, osteocalcin, 25- $(\mathrm{OH}) \mathrm{D}_{3}$, and 1,25-(OH) ${ }_{2} \mathrm{D}_{3}$ values at renal transplant $(0)$ and 12 months later (12).

\begin{tabular}{|c|c|c|c|c|c|c|c|c|}
\hline & \multicolumn{2}{|c|}{ PTH } & \multicolumn{2}{|c|}{ OC } & \multicolumn{2}{|c|}{$25-(\mathrm{OH}) \mathrm{D}_{3}$} & \multicolumn{2}{|c|}{$1,25-(\mathrm{OH})_{2} \mathrm{D}_{3}$} \\
\hline & 0 & 12 & 0 & 12 & 0 & 12 & 0 & 12 \\
\hline All $(N=13)^{a}$ & $249 \pm 163$ & $66 \pm 26^{*}$ & $67 \pm 117$ & $53 \pm 75$ & $22 \pm 15$ & $26 \pm 12$ & $29 \pm 6$ & $36 \pm 10$ \\
\hline Same $(N=3)$ & $180 \pm 139$ & $58 \pm 35$ & $157 \pm 253$ & $37 \pm 34$ & $25 \pm 15$ & $28 \pm 6$ & $27 \pm 5$ & $31 \pm 8$ \\
\hline Better $(\mathrm{N}=3)$ & $432 \pm 190$ & $44 \pm 5$ & $58 \pm 20$ & $17 \pm 14$ & $26 \pm 11$ & $31 \pm 8$ & $30 \pm 9$ & $37 \pm 12$ \\
\hline Recovered $(\mathrm{N}=6)$ & $180 \pm 113$ & $77 \pm 25$ & $32 \pm 24$ & $79 \pm 100$ & $23 \pm 17$ & $32 \pm 24$ & $30 \pm 6$ & $36 \pm 13$ \\
\hline
\end{tabular}

Data are reported as means $\pm \mathrm{SD}$. PTH = serum parathormone (normal range $=8-76 \mathrm{pg} / \mathrm{dL}$ ); OC = serum osteocalcin (normal range $=6-37 \mathrm{pg} / \mathrm{dL}$ ); $25-(\mathrm{OH}) \mathrm{D}_{3}=25$-hydroxyvitamin $\mathrm{D}$ (normal range $=16-74 \mathrm{ng} / \mathrm{mL}$ ); $1,25-(\mathrm{OH})_{2} \mathrm{D}_{3}=1,25$-dihydroxyvitamin $\mathrm{D}$ (normal range $=16-74 \mathrm{pg} / \mathrm{mL}$ ). For group descriptions, see legend to Table 1.

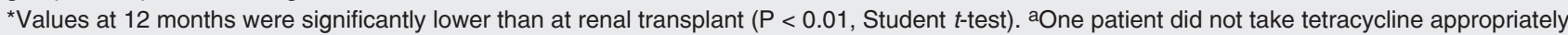
prior to the second biopsy; thus, analysis of improvement at 12 months was done for only 12 patients. 
no changes were seen over the first year. Four of the 13 patients (30\%) presented hypophosphatemia in the first month. However, mean phosphorous levels for the entire group remained within the normal range. Throughout the first year, there were no significant changes in total serum alkaline phosphatase.

Table 2 shows the mean values for PTH and osteocalcin, as well as $1,25-(\mathrm{OH})_{2} \mathrm{D}_{3}$ and $25-(\mathrm{OH}) \mathrm{D}_{3}$, during the study period. Immediately prior to RT, PTH levels were elevated in 10 patients $(77 \%)$ and normal in only 3 (23\%). Soon thereafter, the patients with elevated PTH presented decreases in these levels. At the end of the 3rd month, and through the end of the study, only 3 patients had increased PTH levels, and those increases were slight. Although not statistically significant, mean osteocalcin remained elevated throughout the first month and then normalized by the 6th month. At the end of the first year, mean osteocalcin levels had again increased, but not to statistically significant levels. Serum calcitriol and 25$(\mathrm{OH}) \mathrm{D}_{3}$ levels remained within the normal range throughout the 12-month study period.

\section{Bone mineral densitometry}

BMD was performed, on average, $1.9 \pm$ 1.7 and $12.8 \pm 1.2$ months after RT. The mean interval between the two BMD studies was $10.9 \pm 0.9$ months.

Table 3 and Figure 1 show the BMD data during the first month and at 1 year after transplantation. BMD was decreased in the lumbar vertebrae, but was significantly increased in the total femoral neck.

\section{Histomorphometric analysis of the bone biopsies}

Bone biopsies were obtained approximately $1.4 \pm 0.7$ and $13.4 \pm 1.2$ months after $\mathrm{RT}$. The mean interval between the two bone biopsies was $11.9 \pm 1.4$ months. There were no severe biopsy complications or any documented cases of osteonecrosis or bone fractures during the follow-up period. Table 4 shows the static and dynamic parameters of the bone biopsies obtained at RT and after 1 year. One year after transplantation, there was an increase of near-significance in bone volume. However, both before and after RT, mean values remained within the normal range. After 1 year, the minimal fibrosis observed in the first biopsy had almost entirely disappeared. Similarly, the osteoclas-

Table 3. Bone densitometry at the first month (first measurement) and 1 year after transplantation (second measurement).

\begin{tabular}{lcc}
\hline Bone densitometry sites & First measurement & Second measurement \\
\hline $\mathrm{L} 1-\mathrm{L} 4\left(\mathrm{~g} / \mathrm{cm}^{2}\right)$ & $0.935 \pm 0.109$ & $0.902 \pm 0.099^{\star}$ \\
& $(92.3 \pm 13.7 \%)$ & $(89.6 \pm 11.8 \%)$ \\
Total femoral neck $\left(\mathrm{g} / \mathrm{cm}^{2}\right)$ & $0.817 \pm 0.065$ & $0.853 \pm 0.070^{*}$ \\
& $(86.6 \pm 8.7 \%)$ & $(91.1 \pm 10.7 \%)^{*}$ \\
\hline
\end{tabular}

Data are reported as means \pm SD for $\mathrm{N}=13$ patients in each group and percentage of the age and sex-matched population.

${ }^{*} \mathrm{P}<0.05$ compared to first measurement (Student $t$-test).

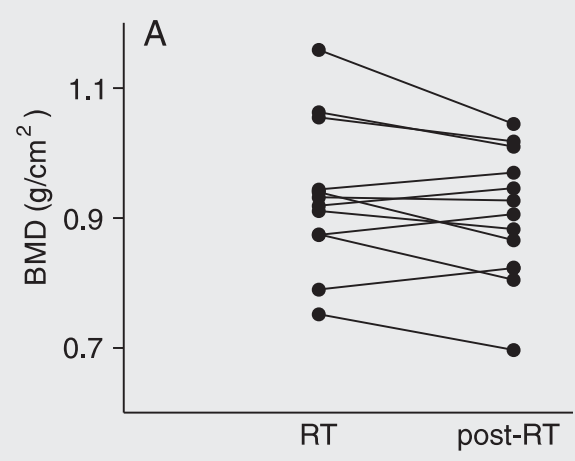

Figure 1. Bone mineral density (BMD) in the lumbar vertebrae (A) and femoral neck (B) measured at the time of renal transplantation (RT) and 1 year later (post-RT). RT and post-RT groups consisted of 13 patients each. 
tic surface had decreased, with the difference almost reaching statistical significance $(\mathrm{P}=0.08)$.

Five patients (38\%) presented mean aluminum bone-surface coverage of $30.9 \pm 15 \%$ $(20.9,51.2,20.5,44.2$, and $17.6 \%)$ at the

Table 4. Histomorphometric analysis of the bone biopsy performed at the time of renal transplantation (first biopsy) and 1 year later (second biopsy).

\begin{tabular}{|c|c|c|c|}
\hline & $\begin{array}{l}\text { First biopsy } \\
\qquad(\mathrm{N}=13)\end{array}$ & $\begin{array}{l}\text { Second biopsy } \\
\qquad(N=12)\end{array}$ & $\begin{array}{l}\text { Normal } \\
\text { range }\end{array}$ \\
\hline Bone volume (\%) & $23.9 \pm 4.7$ & $28.3 \pm 5.8$ & $24.6 \pm 7.1$ \\
\hline Osteoid volume (\%) & $4.1 \pm 2.7$ & $3.4 \pm 2.7$ & $2.3 \pm 2.4$ \\
\hline Osteoid thickness $(\mu \mathrm{m})$ & $8 \pm 2$ & $13.8 \pm 6.2^{*}$ & $11.2 \pm 3.4$ \\
\hline Osteoid surface (\%) & $27.4 \pm 11.3$ & $21.0 \pm 14.8$ & $13.3 \pm 11.1$ \\
\hline Eroded surface (\%) & $5.7 \pm 2.4$ & $5.6 \pm 2.2$ & $2.1 \pm 1.8$ \\
\hline Osteoblast surface (\%) & $4.0 \pm 3.3$ & $3.1 \pm 2.6$ & $1.6 \pm 3.3$ \\
\hline Fibrosis (\%) & $0.2 \pm 0.2$ & $0^{*}$ & 0 \\
\hline Osteoclast surface (\%) & $0.8 \pm 0.8$ & $0.5 \pm 0.4$ & $0.03 \pm 0.09$ \\
\hline Aluminum surface (\%) & $30.9 \pm 15.6$ & $0^{*}$ & 0 \\
\hline Mineral apposition rate ( $\mu /$ day) & nd & $0.5 . \pm 0.2$ & $0.68-1.19$ \\
\hline Mineralization surface (\%) & nd & $8.3 \pm 4.1$ & $13.8 \pm 6.0$ \\
\hline Bone formation rate $\left(\mu \mathrm{m}^{3}\left(\mu \mathrm{m}^{2}\right)^{-1}\right.$ day $\left.^{-1}\right)$ & nd & $0.04 \pm 0.03$ & $0.1 \pm 0.03$ \\
\hline Adjusted apposition rate ( $\mu /$ day) & nd & $0.2 \pm 0.03$ & $0.5 \pm 0.2$ \\
\hline Mineralization lag time (days) & nd & $60 \pm 29.9$ & $23.0 \pm 2.4$ \\
\hline
\end{tabular}

Data are reported as means $\pm \mathrm{SD}$. $\mathrm{nd}=$ not detected.

${ }^{*} \mathrm{P}<0.05$ compared to first biopsy (Student $t$-test).

Figure 2. Role of bone formation rate (BFR) measured by histomorphometry of the bone biopsy obtained at the time of renal transplantation (RT) and 1 year later (post-RT). The 12 patients are indicated by different symbols.

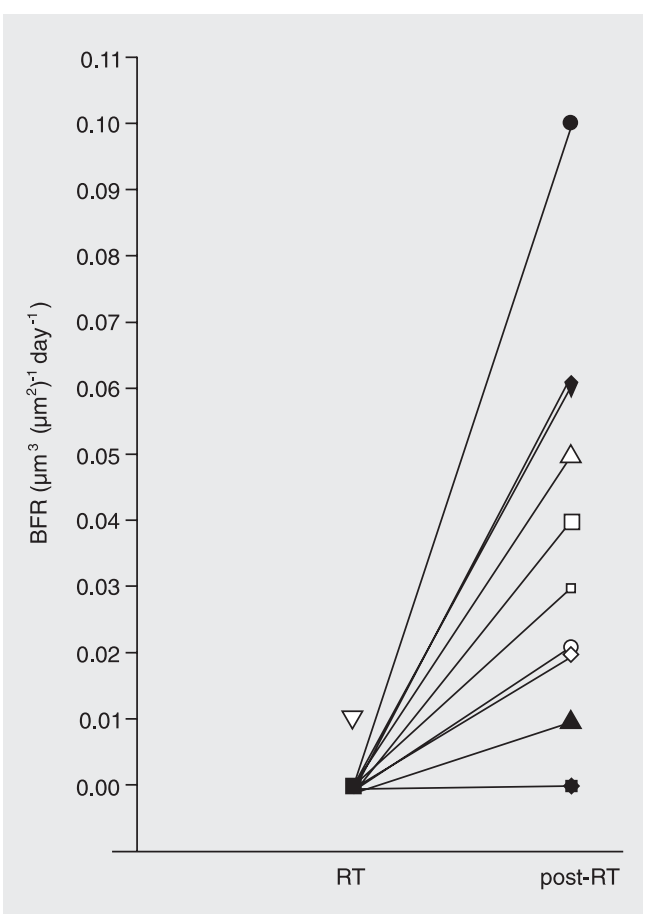

time of the first biopsy. None of the patients presented detectable aluminum staining in the second biopsy.

The bone biopsies obtained at the time of transplantation demonstrated a marked reduction of bone remodeling, with no tetracycline labeling in twelve cases (92\%). One patient had a very low, but measurable, BFR $\left(0.01 \mu \mathrm{m}^{3}\left(\mu \mathrm{m}^{2}\right)^{-1}\right.$ day $\left.^{-1}\right)$ in the first biopsy. Coincidentally, this was the same patient who had not been taking tetracycline appropriately prior to the second biopsy, a fact that interfered with his BFR measurement.

Of the 12 patients who took the appropriate tetracycline doses before the second biopsy, 9 showed a significantly lower surface mineralization. Of these 9, 6 presented a BFR higher than $0.031 \mu \mathrm{m}^{3}\left(\mu \mathrm{m}^{2}\right)^{-1}$ day $^{-1}$ and were therefore considered to have normal bone remodeling at the end of the first posttransplant year, and 3 showed a low, but measurable, BFR $\left(0.02 \pm 0.006 \mu \mathrm{m}^{3}\left(\mu \mathrm{m}^{2}\right)^{-1}\right.$ day $^{-1}$ ). The remaining 3 patients had no detectable tetracycline labeling. Figure 2 shows the BFR values obtained from the first and second bone biopsies.

Neither osteoid volume nor osteoid surface changed after 1 year. However, osteoid thickness increased significantly $(\mathrm{P}=0.02)$.

Of the 12 patients who took tetracycline, 6 had normal biopsies at the end of the first year. Although the other 6 patients still presented features of ABD at that time, 3 of them showed much better dynamic parameters. Therefore, there was an overall improvement of BFR in 9 of the 12 patients.

There were no differences in BFR normalization between patients who had positive aluminum staining in the first biopsy and those who did not. After RT, bone remodeling improved in 3 of the five aluminum-positive patients. Five of the 7 aluminum-negative patients showed similar improvement $(\mathrm{P}=\mathrm{NS})$.

We compared patients whose BFR normalized with those in whom it did not and found no differences between the two groups 
relating to age, presence of diabetes, duration of dialysis, cumulative doses of steroid and cyclosporine (as well as cyclosporine through levels), basal and 12-month serum levels of creatinine, PTH, total serum calcium, phosphorus, serum $25-(\mathrm{OH}) \mathrm{D}_{3}$ or calcitriol. In multiple logistic regression analysis, none of the preceding variables predicted bone remodeling improvement 1 year after RT. Using the same logistic model, none of the static parameters predicted the improvement in BFR. We also divided patients into three groups: those who presented no improvement in BFR (same), those who presented partial reversal of ABD (better) and those in whom ABD was cured (recovered). There were also no differences among these three groups (part of these data are presented in Tables 1 and 2). Finally, of the patients who presented an improvement in BFR, we analyzed those 5 who did not have aluminum intoxication and found no difference between them and the remaining patients in relation to the previously designated variables.

\section{Discussion}

It has been demonstrated that patients with secondary hyperparathyroidism experience bone loss after RT (3). However, little is known about the effect of transplantation on ABD. One study that evaluated low bone turnover patients after kidney transplantation (using bone histology) was performed in patients with severe aluminum intoxication and showed an improvement in bone remodeling 6 months after reversal of uremia (4). Patients with ABD are usually asymptomatic and convincing transplant patients to agree to participate in a study where clear advantages to them are few and two bone biopsies are required is, of course, problematic. These factors contributed to a low enrollment and may account for the under-reporting of the impact of RT on ABD bone remodeling, despite the fact that $\mathrm{ABD}$ is now commonly seen in uremic patients.

In the present study, the first bone biopsy was performed as early as possible after transplantation, taking into consideration the time required for recovery from surgery and for tetracycline labeling. This policy resulted in a mean exposure to oral glucocorticoids of $1.4 \pm 0.7$ months before the first biopsy could be obtained. Long periods of exposure to steroids may reduce BFR. One could argue that this short period of exposure interfered with the initial diagnosis. However, the experience at our bone metabolism laboratory, as well as data in the literature, suggest that it takes more than a few weeks of steroids to reduce BFR $(4,11)$. In addition, another recently performed prospective study evaluated patients with high and low bone turnover before and 6 months after RT (12). Patients were submitted to bone biopsies before RT and, therefore, before starting steroid therapy. These investigators observed that of the $12 \mathrm{ABD}$ patients, 6 showed significant improvement in BFR in the second bone biopsy. Since their results were similar to ours and there was no possibility of steroid interference, we can assume that this drug had little or no influence on our BFR findings.

The present study could provide new insights into the pathogenesis of ABD. The most important finding of our study was that ABD can be a reversible metabolic bone disorder after recuperation of normal renal function. Nine of the 12 patients showed an improvement in bone formation rate in the second biopsy. This implies that ABD is a disease or, at least, a metabolic bone disorder partially related to the uremic environment. Bone formation returned to normal 1 year after RT. This was indicated in all patients by complete clearance of aluminum from the bone mineralizing surface, improvement of bone formation rate and unchanged osteoid volume, as well as by improvement in bone histology in $75 \%$ of patients. These improvements were achieved through RT 
alone. Moreover, none of the 13 patients presented bone fractures, osteonecrosis or symptoms of osteoarticular disease during the 1-year study period.

Post-transplantation metabolic changes, such as hypercalcemia or hypophosphatemia, have been reported in other forms of renal osteodystrophy such as hyperparathyroidism (13). In the present study, we also observed hypophosphatemia in a few cases, but no hypercalcemia. We were unable to detect differences in the recovery of BFR in patients who presented normal phosphorous levels in comparison to those who did not. Nevertheless, it would be interesting to examine the assumption that complete normalization of phosphorous levels could help patients to achieve even greater improvement in bone remodeling. However, the size of the population evaluated in this study was too small to permit such analysis.

The second important finding of our study was that the BFR of patients with $\mathrm{ABD}$, even those with normal PTH levels, returned to normal after reversal of the uremic state. PTH levels were only slightly to moderately increased at transplantation and, in the majority of patients, returned to normal at the end of the third month. At 1 year, bone remodeling had been recovered in $75 \%$ of the patients. This finding is consistent with data reported by Bianchi et al. (14), who studied patients with mild chronic renal insufficiency (creatinine clearance of 36-64 $\mathrm{mL} / \mathrm{min}$ ). Their patients did not develop ABD despite presenting significantly lower PTH levels $(30-99 \mathrm{pg} / \mathrm{mL})$ than ours $(23-710 \mathrm{pg} /$ $\mathrm{mL})$. It is known that high PTH levels are necessary for sustaining bone remodeling during the dialysis period. However, these findings suggest that such high levels may not be required in cases of mild renal insufficiency or normal renal allograft function such as those described here. Since it has been shown that elevated serum phosphorus levels may contribute to bone resistance to $\mathrm{PTH}$, the post-transplant decrease in phos- phorus could help normalize the bone response to this hormone (15).

Also of note is the fact that, during the process of bone remodeling that occurred throughout the study period, no increase was observed in any other bone remodeling marker, such as total serum alkaline phosphatase or osteocalcin. A putative explanation could be the fact that there were only minor changes in histomorphometric bone parameters during recovery. An increase in bone volume and osteoid thickness, as well as a decrease in fibrosis and in the osteoclast covering of the bone surface, were observed. However, these changes, although statistically significant, occurred within the normal range of bone remodeling and might not have been sufficiently pronounced to promote changes in plasma levels of these bone metabolism markers. Regarding $25-(\mathrm{OH}) \mathrm{D}_{3}$ and $1,25-(\mathrm{OH})_{2} \mathrm{D}_{3}$, we can conclude that their post-transplant serum concentrations were appropriate, since there was an improvement in BFR.

Another point which merits consideration is that 1 year after transplantation, $\mathrm{ABD}$ patients in this study presented less than the expected degree of bone mass loss. Our results differ from those of previous studies, which showed a much more pronounced bone mass loss after RT $(1,3)$. However, judging by the PTH levels reported, it is likely that those studies included patients with high-turnover bone disease. Therefore, our results cannot be compared to theirs. In general, it seems that ABD patients experience significantly less RT bone loss than do secondary hyperparathyroidism patients. In contrast, Casez et al. (16) reported an increase in bone mass loss in patients with low serum PTH levels. The low PTH levels suggest that those patients suffered from ABD. Based on this finding, one could argue that patients with $\mathrm{ABD}$ experience greater $\mathrm{BMD}$ decreases than do ADB-free patients. However, the PTH values reported by these investigators were lower than those observed 
in our patients $(140 \pm 24$ vs $249 \pm 163 \mathrm{pg} /$ $\mathrm{mL}$ ) and were measured 1 week after RT (when, typically, renal function has only partially recovered). Therefore, no direct comparison is possible, since we measured PTH levels immediately before RT. Finally, these authors did not perform bone biopsies (16), which could have confirmed whether their patients actually suffered from lowturnover bone disease.

Few studies have described the histology of the bone changes occurring after RT (36). Most investigators have employed bone densitometry in the evaluation of post-transplant bone disease, but it is well known that bone biopsy provides the only reliable information about bone remodeling. In our analysis, the comparison between the subgroup of patients whose remodeling indices normalized and the subgroup whose indices did not, provides no information related to differences in age, time in dialysis, drug dosage, or biochemical markers. With a larger cohort of subjects, we might have found sufficient differences to allow speculation regarding improved treatment techniques. In addition, it is possible that some patients might never recover from their ABD in the years following transplantation. Cueto-Manzano et al. (5) studied 55 RT patients at 10 years post-transplant and found that $23.2 \%$ had ABD.

Aluminum intoxication can prevent recovery from ABD. Since only 4 patients received oral aluminum and received it for only a short period, the dialysis water is the probable source of the aluminum intoxication observed in our patients. When first described, ABD was associated with aluminum intoxication (17). Later, it was demonstrated that $\mathrm{ABD}$ could also occur in the absence of aluminum intoxication (2). This indicates that, although aluminum plays a major role in $\mathrm{ABD}$ development, there are other, equally important factors. Both aspects are documented in the present study. Only $38 \%$ of the first biopsies stained posi- tively for aluminum. Aluminum was completely cleared from the bone by the end of the first year. Neither presence nor absence of aluminum in the first biopsy was a factor in BFR improvement by 1 year after RT and absence of aluminum at that time did not ensure recovery from ABD.

In a previous study on the course of aluminum-related osteomalacia, we demonstrated that aluminum is cleared from the bone after RT and does not jeopardize BFR improvement or recovery of osteoid thickness (4). These findings confirm those of the present study, demonstrating that, even in the presence of low bone activity or total recovery of bone remodeling, aluminum is partially or totally cleared from the bone after successful RT.

Our patients (unlike patients with aluminum-induced osteomalacia or a long-lasting graft) did not experience fractures or osteonecrosis episodes after transplantation, despite weight gain and the use of large doses of steroids, a fact that confirms our bone histomorphometric findings. Since it is known that patients with high-turnover bone disease may experience significant side effects from the use of steroids, our results may lead to speculation as to whether low bone remodeling activity protects against steroid-induced osteoporosis (18). In these high-turnover bone disease patients, steroids may worsen the result of PTH-induced hyperabsorption by inhibiting osteoblastogenesis and thus canceling PTH-induced osteoblastogenesis (19). In ABD patients, even cyclosporine could be beneficial due to its stimulating effect on bone remodeling (20).

An interesting question is whether most ABD patients present BFR recovery after RT and, if so, why so many studies have found a high incidence of ABD in long-term, post-RT patients. For example, Faugere et al. (21) reported a high prevalence of low bone turnover and osteomalacia after RT. Similarly, Parker et al. (22) described a pro- 
gressive reduction in bone turnover which could promote or prolong the adynamic state. However, these were cross-sectional studies performed in mostly symptomatic patients, with various follow-up times. Under the conditions described in the study of Parker et al. (22), it would not be possible to determine the pre-RT pattern of bone turnover. Therefore, our findings cannot be compared to those from these studies. We analyzed the short-term (one-year) course of biopsyproven $\mathrm{ABD}$ after RT. It is well known that the most significant loss of bone mass occurs in the first 2 years post-RT. However, there have been no studies evaluating postRT patient progress for longer periods of time.

In order to preserve bone integrity, many recent studies have used various drug regimens, such as hormone replacement, calcitonin, calcium, or vitamin D (18). Some RT studies have also employed bisphosphonates to prevent post-transplant bone loss, but only one study involved bone biopsies, which were performed in order to evaluate the possible effects of prophylactic administration of pamidronate on bone histology (23). Coco et al. (23) analyzed the effects of pamidronate plus oral calcium and vitamin D (versus those of calcium and vitamin D) on BMD and bone histology. Despite the fact that patients receiving pamidronate presented less BMD loss than that observed in the control group, these investigators reported a significant decrease in cellular elements and suppression of bone turnover in the pamidronate group. According to the authors (23), all patients presented $\mathrm{ABD}$ after pamidronate administration. One question that arises is if we could use this drug in those patients who already have ABD.

Due to the small number of patients studied, it was not possible to identify the potential factors related to BFR recovery. In light of these findings, we conclude that bisphosphonate-based treatment of patients suffering from low-turnover bone disease should be considered with some caution. Perhaps these patients should not receive any kind of special treatment in order to regenerate bone remodeling, at least in the first year after RT. However, further studies are needed in order to confirm these hypotheses.

\section{Acknowledgments}

We would like to thank Jefferson Davis Boyles for translation assistance and Rozidete Bizerra for technical assistance with bone histology.

\section{References}

1. Heaf JG (2003). Bone disease after renal transplantation. Transplantation, 75: 315-325.

2. Salusky IB \& Goodman WG (2001). Adynamic renal osteodystrophy: Is there a problem? Journal of the American Society of Nephrology, 12: 1978-1985.

3. Julian BA, Laskow DA, Dubovisky J et al. (1991). Rapid loss of vertebral mineral density after renal transplantation. New England Journal of Medicine, 325: 544-550.

4. David-Neto E, Jorgetti V, Soeiro NM et al. (1993). Reversal of aluminum-related bone disease after renal transplantation. American Journal of Nephrology, 13: 12-17.

5. Cueto-Manzano AM, Konel S, Hutchison AJ et al. (1999). Bone loss in long-term renal transplantation: histopathology and densitometry analysis. Kidney International, 55: 2021-2029.

6. Rojas E, Carlini R, Clesca P et al. (2003). The pathogenesis of osteodystrophy after renal transplantation as detected by early al- terations in bone remodeling. Kidney International, 63: 1915-1923.

7. Jorgetti V, Soeiro NM, Mendes V et al. (1994). Aluminium-related osteodystrophy and desferrioxamine treatment: role of phosphorus. Nephrology, Dialysis, Transplantation, 9: 668-674.

8. Parfitt AM, Drezner MK, Glorieux FH et al. (1987). Bone histomorphometry: standardization of nomenclature, symbols, and units. Report of the ASBMR Histomorphometry Nomenclature Committee. Journal of Bone and Mineral Research, 2: 595-610.

9. Garcia-Carrasco M, Gruson M, de Vernejoul MC et al. (1988). Osteocalcin and bone morphometric parameters in adults without bone disease. Calcified Tissue International, 42: 13-17.

10. Melsen F \& Mosekilde L (1978). Tetracycline double-labeling of iliac trabecular bone in 41 normal adults. Calcified Tissue Research, 26: 99-102.

11. Lo Cascio V, Kanis JA, Beneton MN et al. (1995). Acute effects of deflazacort and prednisone on rates of mineralization and bone 
formation. Calcified Tissue International, 56: 109-112.

12. Cruz EAS, Lugon JR, Jorgetti V et al. (2003). Histologic evolution of bone disease 6 months after successful kidney transplantation. American Journal of Kidney Diseases, 44: 747-756.

13. Moshe L (2001). Post-transplant hypophosphatemia. Kidney International, 59: 2377-2387.

14. Bianchi ML, Colantonio G, Campanini F et al. (1994). Calcitriol and calcium carbonate therapy in early chronic renal failure. Nephrology, Dialysis, Transplantation, 9: 1595-1599.

15. Bover JA, Trinidad P, Rodriguez M et al. (1999). Dynamics of skeletal resistance to parathyroid hormone in the rat effect of renal failure and dietary phosphorus. Bone, 25: 279-285.

16. Casez JP, Lippuner K, Horber FF et al. (2002). Changes in bone mineral density over 18 months following kidney transplantation: the respective roles of prednisone and parathyroid hormone. Nephrology, Dialysis, Transplantation, 17: 1318-1326.

17. Ott SM, Maloney NA, Coburn JW et al. (1982). The prevalence of bone aluminum deposition in renal osteodystrophy and its relation to the response to calcitriol therapy. New England Journal of Medicine, 307: 709-713.

18. Torres A, Lorenzo V \& Salido E (2002). Calcium metabolism and skeletal problems after transplantation. Journal of the American Society of Nephrology, 13: 551-558.

19. Weinstein RS, Jilka RL, Parfitt AM et al. (1998). Inhibition of osteoblastogenesis and promotion of apoptosis of osteoblasts and osteoclasts by glucocorticoids: Potential mechanisms of their deleterious effects on bone. Journal of Clinical Investigation, 102: 274282.

20. Sprague SM (2000). Mechanism of transplantation-associated bone loss. Pediatric Nephrology, 14: 650-653.

21. Faugere MC, Mawad H, Qi $Q$ et al. (2000). High prevalence of low bone turnover and ocurrence of osteomalacia after kidney transplantation. Journal of the American Society of Nephrology, 11: 10931099.

22. Parker CR, Freemont AJ, Blackwell PJ et al. (1999). Cross-sectional analysis of renal transplantation osteoporosis. Journal of Bone and Mineral Research, 14: 1943-1951.

23. Coco M, Glicklich D, Faugere MC et al. (2003). Prevention of bone loss in renal transplant recipients: a prospective, randomized trial of intravenous pamidronate. Journal of the American Society of $\mathrm{Ne}$ phrology, 14: 2669-2676. 